\title{
On almost continuous functions and peculiar points
}

\author{
Anna Loranty ${ }^{1}$ (D) $\cdot$ Ryszard J. Pawlak ${ }^{1}$ (D) Małgorzata Terepeta ${ }^{2,3}$ \\ Dedicated to Professor Zbigniew Grande on the occasion of his 70th birthday
}

Received: 8 December 2017 / Revised: 10 April 2018 / Accepted: 27 May 2018 / Published online: 26 July 2018 (c) The Author(s) 2018

\begin{abstract}
We introduce the concept of a peculiar point (of the first and second kind), which combines stability of functions around a given point on a large set in the sense of Lebesgue measure with strong chaos of a function (in the sense of its entropy value) around this point. We prove that almost continuity of a function is equivalent to the fact that in every $\Gamma$-neighbourhood of this function one can find a continuous function having a peculiar point either of the first or second kind.
\end{abstract}

Keywords Almost continuity · Graph of a function · Dynamical system · Entropy point · (Strongly) 0-approximate continuity · (Almost) stable point · Odd point . Peculiar point

Mathematics Subject Classification 26A15 $\cdot 26 \mathrm{~A} 18 \cdot 54 \mathrm{C} 70 \cdot 37 \mathrm{~B} 55$

\section{Introduction and preliminaries}

Dynamical systems with discrete time observations have many practical applications in various fields, including economics, biology, information flow, or physics [2,6,12]. This is the reason why for many years properties of functions have been examined in the context of their stability and chaotic behaviour. There are many (but not equiv-

Anna Loranty

anna.loranty@wmii.uni.lodz.pl

Ryszard J. Pawlak

ryszard.pawlak@wmii.uni.lodz.pl

Małgorzata Terepeta

malgorzata.terepeta@p.lodz.pl

1 Faculty of Mathematics and Computer Science, Łódź University, Banacha 22, 90-238 Łódź, Poland

2 Center of Mathematics and Physics, al. Politechniki 11, 90-924 Łódź, Poland

3 Institute of Mathematics, Łódź University of Technology, ul. Wólczańska 215, 90-924 Łódź, Poland 
alent) definitions of chaos (compare [3] and [10]). Therefore it seems interesting to consider the positive entropy of a function as an indicator of chaos. In this paper, we will study local problems of behaviour of functions (around a fixed point). As remarked in the monograph [10] from 2017, such framework is focused on functions mapping the unit interval into itself, which will be the subject of our investigation, as well.

In the real analysis theory, particularly in the research of students of Zbigniew Grande (especially in the habilitation theses of Tomasz Natkaniec and Aleksander Maliszewski), almost continuous functions play the basic role (an almost continuous function has the property that in any open neighbourhood of its graph ( $\Gamma$-neighbourhood) there is a graph of some continuous function). In this paper, we will show that in case of a function acting in the unit interval, in the above definition we may demand that such function has a peculiar point (of the first or second kind). It is worth mentioning, that to investigate such kind of functions, it is necessary to consider entropy in topological terms for discontinuous functions. From this point of view it was some inconvenience that in the literature until recently, the topological entropy was defined for continuous maps while the metric (Shannon) entropy was defined for measurable functions which may be strongly discontinuous. This issue was resolved by Čiklová who in 2005 [1] defined a topological entropy for discontinuous maps (of a compact metric space) with almost all the standard properties.

Throughout the paper we will use the standard notations. $\mathbb{N}$ stands for the set of positive integers, $\mathbb{Q}$ for the set of rational numbers. By $\mathcal{L}$ we denote the $\sigma$-algebra of all Lebesgue measurable sets and $\ell$ is the Lebesgue measure. Let $\mathbb{I}=[0,1], \mathbb{I}^{2}=\mathbb{I} \times \mathbb{I}$. The symbol $\operatorname{proj}_{X}(A)$ will stand for a projection of a set $A \subset \mathbb{I}^{2}$ on $O x$. For a function $f: \mathbb{I} \rightarrow \mathbb{I}$ by $f^{n}$ we denote the $n^{\text {th }}$ iteration of $f$, that means $f^{n}(x)=f\left(f^{n-1}(x)\right)$ and $f^{0}(x)=x$ for $x \in \mathbb{I}$ and $n \in \mathbb{N}$. We put Fix $(f)$ for the set of all fixed points of $f, \Gamma(f)$ for the graph of $f$ and $\mathfrak{D}$ for the family of all Darboux functions.

Let $f: \mathbb{I} \rightarrow \mathbb{I}, A \subset \mathbb{I}, n \in \mathbb{N}$ and $\varepsilon>0$. A set $S \subset A$ is called $(f, A, \varepsilon, n)$ separated if for all $x, y \in S, x \neq y$, one can find a positive integer $i$ such that $0 \leqslant i<n$ and $\left|f^{i}(x)-f^{i}(y)\right|>\varepsilon$. Let $s(f, A, \varepsilon, n)$ denote the cardinality of an $(f, A, \varepsilon, n)$-separated set with the maximal possible number of points. The topological entropy of a function $f$ on the set $A$ is the number

$$
h(f, A)=\lim _{\varepsilon \rightarrow 0} \limsup _{n \rightarrow \infty} \frac{1}{n} \log s(f, A, \varepsilon, n) .
$$

If $A=\mathbb{I}$, then we shortly write $h(f)$ instead of $h(f, \mathbb{I})$.

In the next considerations we will need the following results.

Lemma 1.1 ([5]) Let $f: \mathbb{I} \rightarrow \mathbb{I}, Y \subset \mathbb{I}$ and $W_{1}, W_{2} \subset Y$ be closed and disjoint sets such that $W_{i} \underset{f}{\rightarrow} W_{j}$ (i.e. $W_{j} \subset f\left(W_{i}\right)$ ), for $i, j \in\{1,2\}$. Then $h(f, Y)>0$.

Lemma 1.2 Let $P \subset \mathbb{I}$ be a closed non-degenerate interval, $f: \mathbb{I} \rightarrow \mathbb{I}$ and $[a, b] \subset P$. If there exists $n_{0} \in \mathbb{N}$ such that $f^{n_{0}}([a, b])=P$, then $h(f,[a, b])=h(f, P)$ for $h(f, P) \in[0,+\infty]$. 
Proof For arbitrary $\varepsilon>0$ and $n \in \mathbb{N}$, let $E_{\varepsilon, n} \subset P$ be an $(f, P, \varepsilon, n)$-separated set of the biggest cardinality. Then

$$
h(f, P)=\lim _{\varepsilon \rightarrow 0} \limsup _{n \rightarrow \infty} \frac{1}{n} \log \operatorname{card}\left(E_{\varepsilon, n}\right) .
$$

For fixed $\varepsilon>0$ and $n \in \mathbb{N}$ we assume that

$$
\text { for any } x \in E_{\varepsilon, n}, t_{x} \in[a, b] \text { is a fixed point for which } f^{n_{0}}\left(t_{x}\right)=x \text {. }
$$

It is easy to show that

$$
\text { if } x, y \in E_{\varepsilon, n} \text { and } x \neq y \text {, then } t_{x} \neq t_{y} \text {. }
$$

Consider the set $E_{\varepsilon, n}^{*}=\left\{t_{x} \in[a, b]: x \in E_{\varepsilon, n}\right\}$. From (2) and (1) we have $\operatorname{card}\left(E_{\varepsilon, n}^{*}\right)=\operatorname{card}\left(E_{\varepsilon, n}\right)$. We will show that

$$
E_{\varepsilon, n}^{*} \text { is an }\left(f,[a, b], \varepsilon, n+n_{0}\right) \text {-separated set. }
$$

Obviously, $E_{\varepsilon, n}^{*} \subset[a, b]$. Let $t_{1}, t_{2} \in E_{\varepsilon, n}^{*}$ and $t_{1} \neq t_{2}$. Then, there exist $x_{1}, x_{2} \in E_{\varepsilon, n}$ such that $x_{1}=f^{n_{0}}\left(t_{1}\right)$ and $x_{2}=f^{n_{0}}\left(t_{2}\right)$. From (1) it follows that $x_{1} \neq x_{2}$.

Since $E_{\varepsilon, n}$ is an $(f, P, \varepsilon, n)$-separated set, there exists $0 \leqslant i_{0}<n$ such that $\left|f^{i_{0}}\left(x_{1}\right)-f^{i_{0}}\left(x_{2}\right)\right|>\varepsilon$. Put $j=i_{0}+n_{0}$. Then $0 \leqslant j<n+n_{0}$ and hence $\left|f^{j}\left(t_{1}\right)-f^{j}\left(t_{2}\right)\right|=\left|f^{i_{0}+n_{0}}\left(t_{1}\right)-f^{i_{0}+n_{0}}\left(t_{2}\right)\right|=\left|f^{i_{0}}\left(f^{n_{0}}\left(t_{1}\right)\right)-f^{i_{0}}\left(f^{n_{0}}\left(t_{2}\right)\right)\right|=$ $\left|f^{i_{0}}\left(x_{1}\right)-f^{i_{0}}\left(x_{2}\right)\right|>\varepsilon$, which implies that $E_{\varepsilon, n}^{*}$ is an $\left(f,[a, b], \varepsilon, n+n_{0}\right)$-separated set.

In consequence, we obtain that $s\left(f,[a, b], \varepsilon, n+n_{0}\right) \geqslant \operatorname{card}\left(E_{\varepsilon, n}^{*}\right)=\operatorname{card}\left(E_{\varepsilon, n}\right)$ for any $\varepsilon>0$ and $n \in \mathbb{N}$. Therefore,

$$
\begin{aligned}
\limsup _{n \rightarrow \infty} \frac{1}{n} \log s(f, P, \varepsilon, n) & \geqslant \limsup _{n \rightarrow \infty} \frac{1}{n} \log s(f,[a, b], \varepsilon, n) \\
& \geqslant \limsup _{n \rightarrow \infty} \frac{1}{n+n_{0}} \log \operatorname{card}\left(E_{\varepsilon, n}\right) \\
& =\lim _{n \rightarrow \infty} \frac{n}{n+n_{0}} \limsup _{n \rightarrow \infty} \frac{1}{n} \log \operatorname{card}\left(E_{\varepsilon, n}\right)=h(f, P) .
\end{aligned}
$$

If $h(f, P) \in(0,+\infty)$, from (3) we immediately obtain $h(f,[a, b]) \geqslant h(f, P) / 2$. If $h(f, P)=0$ or $h(f, P)=\infty$ then again from (3) we have $h(f,[a, b])=0$ or $h(f,[a, b])=\infty$ and finally $h(f,[a, b])=h(f, P)$.

\section{Peculiar points and almost continuity}

In the next part of the paper, we will need the notion of an entropy of a function at a point, considered in [4,9]. For a function $f$ a pair $B_{f}=(\mathcal{F}, J)$ is called an $f$-bundle if $\mathcal{F}$ is a family of pairwise disjoint non-singleton continua in $\mathbb{I}$ and $J \subset \mathbb{I}$ is a connected 
set such that $J \subset f(A)$ for any $A \in \mathcal{F}$. If we additionally assume that $A \subset J$ for all $A \in \mathcal{F}$ then $B_{f}=(\mathcal{F}, J)$ will be called an $f$-bundle with dominating fibre.

Similarly, as in the case of an entropy of a function we define a separated set connected with an $f$-bundle. Let $\varepsilon>0, n \in \mathbb{N}, B_{f}=(\mathcal{F}, J)$ be an $f$-bundle and $M \subset \bigcup \mathcal{F}$. We shall say that $M$ is $\left(B_{f}, n, \varepsilon\right)$-separated if for each $x, y \in M, x \neq y$, there is $i \in\{0,1, \ldots, n-1\}$ such that $f^{i}(x), f^{i}(y) \in J$ and $\left|f^{i}(x)-f^{i}(y)\right|>\varepsilon$. Put $s\left(B_{f}, n, \varepsilon\right)=\max \left\{\operatorname{card}(M): M \subset \mathbb{I}\right.$ is a $\left(B_{f}, n, \varepsilon\right)$-separated set $\}$. The number

$$
h\left(B_{f}\right)=\lim _{\varepsilon \rightarrow 0} \limsup _{n \rightarrow \infty} \frac{1}{n} \log s\left(B_{f}, n, \varepsilon\right)
$$

is called an entropy of an $f$-bundle $B_{f}$. It is worth adding that if $B_{f}=(\mathcal{F}, J)$ is an $f$-bundle with dominating fibre and $\mathcal{F}$ is finite then $h\left(B_{f}\right) \geqslant \log \operatorname{card}(\mathcal{F})$ [9].

We shall say that a sequence of $f$-bundles $B_{f}^{k}=\left(\mathcal{F}_{k}, J_{k}\right)$ converges to a point $x_{0}$ (in short $B_{f}^{n} \underset{n \rightarrow \infty}{\longrightarrow} x_{0}$ ), if for any $\varepsilon>0$ there exists $k_{0} \in \mathbb{N}$ such that $\bigcup \mathcal{F}_{k} \subset$ $\left(x_{0}-\varepsilon, x_{0}+\varepsilon\right)$ and $\left(f\left(x_{0}\right)-\varepsilon, f\left(x_{0}\right)+\varepsilon\right) \cap J_{k} \neq \varnothing$ for any $k \geqslant k_{0}$. An entropy of a function $f$ at $x_{0} \in \mathbb{I}[4]$ is the number

$$
e_{f}\left(x_{0}\right)=\sup \left\{\limsup _{n \rightarrow \infty} h\left(B_{f}^{n}\right): B_{f}^{n} \underset{n \rightarrow \infty}{\longrightarrow} x_{0}\right\}
$$

Let $x_{0} \in(0,1)$ and $\left\{a_{n}\right\}_{n \in \mathbb{N}},\left\{b_{n}\right\}_{n \in \mathbb{N}},\left\{c_{n}\right\}_{n \in \mathbb{N}},\left\{d_{n}\right\}_{n \in \mathbb{N}} \subset \mathbb{I}$ be sequences such that $\lim _{n \rightarrow \infty} b_{n}=\lim _{n \rightarrow \infty} c_{n}=x_{0}$ and $c_{n}<d_{n}<c_{n+1}$ and $b_{n+1}<a_{n}<b_{n}$ for any $n \in \mathbb{N}$. An interval set at a point $x_{0}$ is the set given by the following formula:

$$
\bigcup_{n=1}^{\infty} L_{n} \cup\left\{x_{0}\right\} \cup \bigcup_{n=1}^{\infty} R_{n}
$$

where $L_{n}=\left[c_{n}, d_{n}\right]$ and $R_{n}=\left[a_{n}, b_{n}\right]$ for $n \in \mathbb{N}$. Similarly, we define a right-(left-) hand interval set at a point $x_{0} \in \mathbb{I}$. To shorten the notation, for $x_{0}=0$ or $x_{0}=1$ an interval set at a point $x_{0}$ we will be a right-hand or a left-hand interval set at this point, respectively. Let $\mathcal{L}_{i}\left(x_{0}\right)$ denote the set of all interval sets at a point $x_{0}$.

For any $x_{0} \in \mathbb{I}$ and $A \in \mathcal{L}$ if there exists the limit

$$
\lim _{h \rightarrow 0^{+}} \frac{\ell\left(A \cap\left[x_{0}-h, x_{0}+h\right]\right)}{2 h},
$$

then we call it the density of a set $A$ at a point $x_{0}$. Similarly, we define the right(left-)hand density of a set at a point. If $x_{0}=0$ or $x_{0}=1$ then we consider suitable one-sided density of this set at $x_{0}$. If the density of $A$ at a point $x_{0}$ is equal to 1 , then we say that $x_{0}$ is a density point of a set $A$.

We shall say that $f$ is strongly 0-approximately continuous at $x_{0} \in \mathbb{I}$ if there exists a set $A \in \mathcal{L}_{i}\left(x_{0}\right)$ such that $x_{0}$ is a density point of $A, \lim _{A \ni x \rightarrow x_{0}} f(x)=f\left(x_{0}\right)$ and $h(f, A)=0$. 
It is easy to present an example of a function which is strongly 0-approximately continuous at any point from $\mathbb{I}$ (the constant function has this property). Simultaneously, the function from [5, Theorem 2.4] is an example of a function which is not strongly 0 -approximately continuous at any point from $\mathbb{I}$. One can ask, whether there exists a continuous function with such a property.

Let $f: \mathbb{I} \rightarrow \mathbb{I}$ be the tent map. Obviously, $h(f)=\log 2$ (see [10, p. 94]). Moreover, for any non-degenerate interval $[a, b] \subset \mathbb{I}$ one can find the number $n_{0} \in \mathbb{N}$ for which $f^{n_{0}}([a, b])=\mathbb{I}$. Indeed, without loss of generality we may assume that $[a, b] \neq \mathbb{I}$. Then there exist $n_{1} \in \mathbb{N}$ and $i_{1} \in\left\{1,2, \ldots, 2^{n_{1}}\right\}$ such that

$$
\left[\frac{i_{1}-1}{2^{n_{1}}}, \frac{i_{1}}{2^{n_{1}}}\right] \subset[a, b] .
$$

From the definition of the tent map it follows that $f^{n}\left(\left[i_{1}-1 / 2^{n_{1}}, i_{1} / 2^{n_{1}}\right]\right)=\mathbb{I}$ for any $n>n_{1}$. Let $n_{0}=n_{1}+1$. Since $f^{n_{0}}\left(\left[i_{1}-1 / 2^{n_{1}}, i_{1} / 2^{n_{1}}\right]\right) \subset f^{n_{0}}([a, b])$, we obtain $f^{n_{0}}([a, b])=\mathbb{I}$. From Lemma 1.2 it follows that $h(f,[a, b])>0$ for any interval $[a, b] \subset \mathbb{I}$. Hence, we immediately obtain that $f$ is not strongly 0 -approximately continuous at any point from $\mathbb{I}$. Indeed, assume that $f$ is strongly 0 -approximately continuous at $x_{0}$. Then there exists an interval set $A \in \mathcal{L}_{i}\left(x_{0}\right)$ such that $h(f, A)=0$. On the other hand, there exists a closed non-degenerate interval $[a, b] \subset A$ for which $h(f, A) \geqslant h(f,[a, b])>0$, which is a contradiction.

In the paper [5] there were considered functions 0-approximately continuous at a point. We shall say that $f$ is 0-approximately continuous at $x_{0} \in \mathbb{I}$ if there exists a set $A \in \mathcal{L}$ such that $x_{0}$ is a density point of $A, \lim _{A \ni x \rightarrow x_{0}} f(x)=f\left(x_{0}\right)$ and $h(f, A)=0$. Obviously, if a function $f$ is strongly 0 -approximately continuous at a point $x_{0}$, then it is 0 -approximately continuous at this point. The inverse implication does not hold. Indeed, the characteristic function of the set $\mathbb{I} \backslash \mathbb{Q}$ is 0 -approximately continuous at any point from $\mathbb{I} \backslash \mathbb{Q}$ and it is not strongly 0-approximately continuous at this point.

In the paper [8] there was introduced the notion of an odd point for dynamical systems. For a function $f: \mathbb{I} \rightarrow \mathbb{I}$ this definition is as follows. We shall say that $x_{0} \in \mathbb{I}$ is an odd point of a function $f$ if $x_{0}$ is an almost stable point of $f$ (i.e. $x_{0} \in \operatorname{Fix}(f)$ and for any $\varepsilon>0$ there are $\delta>0$ and $i_{0} \in \mathbb{N}$ such that for each $i \geqslant i_{0}$ and $x \in \mathbb{I}$ if $\left|x-x_{0}\right|<\delta$ then $\left|f^{i}(x)-x_{0}\right|<\varepsilon$ ) and an entropy of $f$ at the point $x_{0}$ is infinite. Evidently, if $f$ is a continuous function and $x_{0}$ is an odd point of $f$, then $x_{0}$ is a stable point of $f$. It means $x_{0} \in \operatorname{Fix}(f)$ and for any $\varepsilon>0$ there is $\delta>0$ such that for each $i \in \mathbb{N}$ and $x \in \mathbb{I}$ if $\left|x-x_{0}\right|<\delta$ then $\left|f^{i}(x)-x_{0}\right|<\varepsilon$ (see [8]).

In another paper [5] there were considered functions attracting positive entropy at a point. Let $f \in \mathfrak{D}$ and $x_{0} \in \mathbb{I}$. We say that $f$ attracts positive entropy at a point $x_{0}$ if for any $\varepsilon>0$ there exists $\delta>0$ such that for each function $g \in B_{\rho_{u}}(f, \delta) \cap \mathfrak{D}$ we have $h\left(g,\left(x_{0}-\varepsilon, x_{0}+\varepsilon\right) \cap \mathbb{I}\right)>0$, where $B_{\rho_{u}}(f, \delta)$ is the open ball with center at $f$ and radius $\delta$ with respect to the metric of uniform convergence.

Definition 2.1 Let $f: \mathbb{I} \rightarrow \mathbb{I}$ be a Darboux function. We say that $x_{0}$ is a peculiar point of the first kind of $f$ if 
(A1) $x_{0}$ is an odd point of $f$;

(B1) $f$ is 0 -approximately continuous at $x_{0}$ and it is not strongly 0 -approximately continuous at $x_{0}$;

(C1) $f$ attracts positive entropy at $x_{0}$.

If in this definition condition (B1) is replaced by the condition

$\left(\mathrm{B} 1^{\prime}\right) f$ is strongly 0 -approximately continuous at $x_{0}$,

then we obtain the definition of a peculiar point of the second kind.

The notion of a peculiar point seems to be very complex. As the name of such a point indicates, it describes a very special situation, when around a point a function is stable on a big set (in the sense of Lebesgue measure) and, simultaneously, it is not only strongly chaotic, but there takes place the so-called entropy black hole phenomenon as well: if another function is close to a function possessing a peculiar point, then its entropy is positive in any neighbourhood of this point (many mathematicians identify this with the chaotic behaviour of a function). So it is natural to ask, if having a peculiar point is a random, incidental property of a function or it is frequent (in some sense). The same question can be posted for continuous functions.

As it was mentioned earlier, in the real analysis theory, almost continuous functions play a very important role. This notion was introduced in 1959 by Stallings in [11]. The main theorem of this work shows that any $\Gamma$-neighbourhood of an almost continuous function contains a continuous function with a peculiar point (of the first or second kind).

Recall the definition of an almost continuous function: we say that a function $f: \mathbb{I} \rightarrow \mathbb{I}$ is almost continuous (in the sense of Stallings) if every open set $U \subset \mathbb{I}^{2}$ containing $\Gamma(f)$ (i.e. a $\Gamma$-neighbourhood of $f$ ) contains the graph of some continuous function $g: \mathbb{I} \rightarrow \mathbb{I}$.

Theorem 2.2 Let $f: \mathbb{I} \rightarrow \mathbb{I}$. The following conditions are equivalent:

(i) $f$ is an almost continuous function.

(ii) Every open set $U \subset \mathbb{I}^{2}$ containing $\Gamma(f)$ contains the graph of some continuous function having a peculiar point of the first kind.

(iii) Every open set $U \subset \mathbb{I}^{2}$ containing $\Gamma(f)$ contains the graph of some continuous function having a peculiar point of the second kind.

Proof The implication ((ii) $\vee($ iii $)) \Rightarrow$ (i) is evident, so we will prove only implications (i) $\Rightarrow$ (ii) and (i) $\Rightarrow$ (iii).

Assume that $f$ is almost continuous and $U \subset \mathbb{I}^{2}$ is an open set containing $\Gamma(f)$. Then there exists a continuous function $\phi: \mathbb{I} \rightarrow \mathbb{I}$ such that $\Gamma(\phi) \subset U$. Clearly, this function has a fixed point $x_{0}$. Without loss of generality we may assume that $x_{0} \in(0,1)$. Let $\varepsilon>0$ be a number such that the square $K=\left[x_{0}-\varepsilon, x_{0}+\varepsilon\right] \times\left[x_{0}-\varepsilon, x_{0}+\varepsilon\right]$ is a subset of $U$. Fix a smaller square $K_{0}$ with center at $x_{0}$ and the edges parallel to the coordinate axes such that $K_{0} \subset K$.

Firstly we will prove that (i) $\Rightarrow$ (ii). On the interval $\operatorname{proj}_{X}\left(K_{0}\right)$ we may consider the classical construction of a Cantor-type set $C$ of positive measure. Clearly, the ends of $\operatorname{proj}_{X}\left(K_{0}\right)$ belong to $C$. Moreover, in the $k^{\text {th }}$ stage of construction we remove $2^{k-1}$ open middle intervals denoted by $\left(a_{k, j}, b_{k, j}\right), j=1,2, \ldots, 2^{k-1}$, respectively. 
We will define a continuous function $\tau: \mathbb{I} \rightarrow \mathbb{I}$ which fulfils conditions from (ii). For any $n \in \mathbb{N}$ and $j \in\left\{1,2, \ldots, 2^{n-1}\right\}$ let us consider the interval $I_{n, j}=\left[a_{n, j}, b_{n, j}\right]$. Let $c_{1}^{n, j}, c_{2}^{n, j}, \ldots, c_{2^{n}}^{n, j}$ be the points from $\left(a_{n, j}, b_{n, j}\right)$ which divide the interval $I_{n, j}$ into $2^{n}+1$ closed intervals of equal length. Put

$$
\tau(x)= \begin{cases}a_{n, k} & \text { for } x=c_{2 m}^{n, k}, n \in \mathbb{N}, k=1,2, \ldots, 2^{n-1}, m=1,2, \ldots, 2^{n-1}, \\ b_{n, k} & \text { for } x=c_{2 m-1}^{n, k}, n \in \mathbb{N}, k=1,2, \ldots, 2^{n-1}, m=1,2, \ldots, 2^{n-1}, \\ x & \text { for } x \in C, \\ \text { linear } & \text { on }\left[a_{n, k}, c_{1}^{n, k}\right],\left[c_{2^{n}}^{n, k}, b_{n, k}\right],\left[c_{i}^{n, k}, c_{i+1}^{n, k}\right], n \in \mathbb{N}, \\ & k=1, \ldots, 2^{n-1}, i=1, \ldots, 2^{n}-1 .\end{cases}
$$

In this way we have defined $\tau$ on the interval $\operatorname{proj}_{X}\left(K_{0}\right)$. We put $\tau(x)=\phi(x)$ on $\mathbb{I} \backslash \operatorname{proj}_{X}(K)$ and linear on $\operatorname{proj}_{X}(K) \backslash \operatorname{proj}_{X}\left(K_{0}\right)$ so that $\tau$ is continuous on the interval $\mathbb{I}$. It is easy to see that $\Gamma(\tau) \subset U$. We will show that this function has desired properties.

Let $\Phi(C)$ be the set of all density points of the set $C$. By the Lebesgue Density Theorem (see [7]) we infer that $\Phi(C) \cap C \neq \varnothing$. Let $y_{0} \in \Phi(C) \cap C$ and obviously, it is not the end of any interval from the complement of $C$ (because if so, then $y_{0}$ is not a density point of $C$ ). The point $y_{0}$ belongs to $C$, hence from the definition of the function $\tau$ we obtain $y_{0} \in \operatorname{Fix}(\tau)$. We shall show that $y_{0}$ is a peculiar point of the first kind, so it fulfils conditions (A1), (B1) and (C1).

To see (A1), observe that for any $\sigma>0$ one can find a neighbourhood $W$ of $y_{0}$ such that $W \subset\left(y_{0}-\sigma, y_{0}+\sigma\right)$ and the ends of $W$ belong to $C$ and they do not belong to the closure of any component of the complement of $C$. Then $\tau^{n}(W)=W$ for any $n \in \mathbb{N}$. Therefore, $y_{0}$ is a stable point of $\tau$ and, in consequence, it is an almost stable point of $\tau$.

Obviously, there is a strictly increasing sequence $\left\{b_{n, k_{n}}\right\}_{n \in \mathbb{N}}$ of the ends of intervals from $\mathbb{I} \backslash C$ such that $\lim _{n \rightarrow \infty} b_{n, k_{n}}=y_{0}$. Put $\mathcal{F}_{n}=\left\{\left[c_{2 i-1}^{n, k_{n}}, c_{2 i}^{n, k_{n}}\right]: i=1, \ldots, 2^{n-1}\right\}$ and $J_{n}=\left[a_{n, k_{n}}, b_{n, k_{n}}\right]$. It is easy to see that $B_{\tau}^{n}=\left(\mathcal{F}_{n}, J_{n}\right)$ is a $\tau$-bundle with dominating fibre. Since $y_{0} \in \operatorname{Fix}(\tau)$ one may easily show that $B_{\tau}^{n} \underset{n \rightarrow \infty}{\longrightarrow} y_{0}$. Moreover, $h\left(B_{\tau}^{n}\right) \geqslant \log 2^{n-1}$. In consequence we obtain that $\limsup _{n \rightarrow \infty} h\left(B_{\tau}^{n}\right)=+\infty$ so $e_{\tau}\left(y_{0}\right)=+\infty$. Finally, $y_{0}$ is an odd point of $\tau$.

To show (B1) we prove first that $\tau$ is 0 -approximately continuous at $y_{0}$. Evidently, $y_{0}$ is a density point of $C$ and $\lim _{C \ni x \rightarrow y_{0}} \tau(x)=\lim _{C \ni x \rightarrow y_{0}} x=y_{0}=\tau\left(y_{0}\right)$. Moreover, since $\tau$ is the identity function on $C$, it is easy to see that $h(\tau, C)=0$. Indeed, take $\varepsilon>0$. For any $n \in \mathbb{N}$, if $M$ is a $(\tau, C, \varepsilon, n)$-separated set, then $\operatorname{card}(M) \leqslant[1 / \varepsilon]+1$ (where $[1 / \varepsilon]$ means the integer part of $1 / \varepsilon$ ). To see that we observe first that for $x \in M \subset C \subset \mathbb{I}$ and any $i \in\{0,1, \ldots, n-1\}$ we have $\tau^{i}(x)=x$. Then, if $\operatorname{card}(M)>[1 / \varepsilon]+1$, we could find two different points $x, y \in M$ such that $|x-y|<\varepsilon$. Hence $\left|\tau^{i}(x)-\tau^{i}(y)\right|=|x-y|<$ $\varepsilon$ for any $i \in\{0,1, \ldots, n-1\}$, which is impossible. Finally, $s(\tau, C, \varepsilon, n) \leqslant$ $[1 / \varepsilon]+1$. Therefore, $h(\tau, C) \leqslant \lim _{\varepsilon \rightarrow 0} \lim \sup _{n \rightarrow \infty} 1 / n \cdot \log ([1 / \varepsilon]+1)=0$, and consequently $h(\tau, C)=0$, which means that $\tau$ is 0 -approximately continuous at $y_{0}$. 
The function $\tau$ is not strongly 0 -approximately continuous at $y_{0}$. Assume contrary, that $\tau$ is strongly 0 -approximately continuous at $y_{0}$. Then there exists an interval set $A \in \mathcal{L}_{i}\left(y_{0}\right)$ for which $h(\tau, A)=0$. Let $(a, b) \subset A \cap(\inf (C), \sup (C))$ be a non-empty interval. Since $C$ is nowhere dense, then there is a non-empty interval $[c, d] \subset(a, b)$ such that $[c, d] \cap C=\varnothing$. Clearly, one can find $n_{0} \in \mathbb{N}$ and $k_{0} \in\left\{1, \ldots, 2^{n_{0}-1}\right\}$ such that $n_{0}>1$ and $[c, d] \subset\left[a_{n_{0}, k_{0}}, b_{n_{0}, k_{0}}\right]$. Analogously, as it was presented for the tent map one may show that there is $n_{1} \in \mathbb{N}$ for which $\tau^{n_{1}}([c, d])=\left[a_{n_{0}, k_{0}}, b_{n_{0}, k_{0}}\right]$. Observe, that by putting $W_{1}=\left[a_{n_{0}, k_{0}}, c_{1}^{n_{0}, k_{0}}\right]$ and $W_{2}=\left[c_{2^{n_{0}}}^{n_{0}, k_{0}}, b_{n_{0}, k_{0}}\right]$ we obtain $W_{i} \rightarrow W_{j}$ for $i, j \in\{1,2\}$ and $W_{1} \cap W_{2}=\varnothing$. From Lemma 1.1, we have $h\left(\tau,\left[a_{n_{0}, k_{0}}, b_{n_{0}, k_{0}}\right]\right)>0$. In consequence, Lemma 1.2 implicates that $h(\tau,[c, d])>0$, which is a contradiction with $h(\tau,[c, d]) \leqslant h(\tau, A)$. Finally, $\tau$ is not strongly 0 -approximately continuous at $y_{0}$.

Now we shall show condition $(\mathrm{C} 1)$ so we shall prove that $\tau$ attracts positive entropy at $y_{0}$. Clearly $\tau \in \mathfrak{D}$.

Let $\varepsilon>0$. Obviously, one can find $n_{0} \geqslant 3$ and $k_{0} \in\left\{1, \ldots, 2^{n_{0}-1}\right\}$ such that $\left(a_{n_{0}, k_{0}}, b_{n_{0}, k_{0}}\right)$ is a component of the complement of $C$ such that $\left[a_{n_{0}, k_{0}}, b_{n_{0}, k_{0}}\right] \subset$ $\left(y_{0}, y_{0}+\varepsilon\right)$. Put $\delta=c_{1}^{n_{0}, k_{0}}-a_{n_{0}, k_{0}}$ and consider $W_{1}=\left[c_{2}^{n_{0}, k_{0}}, c_{3}^{n_{0}, k_{0}}\right]$ and $W_{2}=$ $\left[c_{4}^{n_{0}, k_{0}}, c_{5}^{n_{0}, k_{0}}\right]$. Obviously, $W_{1}, W_{2}$ are disjoint closed sets and $W_{1} \cup W_{2} \subset\left(a_{n_{0}, k_{0}}+\right.$ $\left.\delta, b_{n_{0}, k_{0}}-\delta\right) \subset\left(y_{0}-\varepsilon, y_{0}+\varepsilon\right) \cap \mathbb{I}$.

Let $g \in B(\tau, \delta) \cap \mathfrak{D}$. From the definition of $\tau$ it follows that $\tau\left(c_{3}^{n_{0}, k_{0}}\right)=\tau\left(c_{5}^{n_{0}, k_{0}}\right)=$ $b_{n_{0}, k_{0}}$ and $\tau\left(c_{2}^{n_{0}, k_{0}}\right)=\tau\left(c_{4}^{n_{0}, k_{0}}\right)=a_{n_{0}, k_{0}}$. Then $g\left(c_{2}^{n_{0}, k_{0}}\right) \leqslant a_{n_{0}, k_{0}}+\delta, g\left(c_{4}^{n_{0}, k_{0}}\right) \leqslant$ $a_{n_{0}, k_{0}}+\delta, g\left(c_{3}^{n_{0}, k_{0}}\right) \geqslant b_{n_{0}, k_{0}}-\delta$ and $g\left(c_{5}^{n_{0}, k_{0}}\right) \geqslant b_{n_{0}, k_{0}}-\delta$. From the Darboux property it follows that $g\left(W_{1}\right)=g\left(\left[c_{2}^{n_{0}, k_{0}}, c_{3}^{n_{0}, k_{0}}\right]\right) \supset\left[a_{n_{0}, k_{0}}+\delta, b_{n_{0}, k_{0}}-\delta\right]$ and $g\left(W_{2}\right)=g\left(\left[c_{4}^{n_{0}, k_{0}}, c_{5}^{n_{0}, k_{0}}\right]\right) \supset\left[a_{n_{0}, k_{0}}+\delta, b_{n_{0}, k_{0}}-\delta\right]$. Thus $W_{i} \underset{g}{\rightarrow} W_{j}$ for $i, j \in\{1,2\}$. Lemma 1.1 implies that $h\left(g,\left(y_{0}-\varepsilon, y_{0}+\varepsilon\right) \cap \mathbb{I}\right)>0$ and $\tau$ attracts positive entropy at $y_{0}$. This finishes the proof of implication (i) $\Rightarrow$ (ii).

Now we will prove the implication (i) $\Rightarrow$ (iii). Denote the interval $\operatorname{proj}_{X}\left(K_{0}\right)$ by $[\alpha, \beta]$ and fix $x_{0} \in(\alpha, \beta)$. Let $\left\{a_{n}\right\}_{n \in \mathbb{N}},\left\{b_{n}\right\}_{n \in \mathbb{N}},\left\{r_{n}\right\}_{n \in \mathbb{N}},\left\{s_{n}\right\}_{n \in \mathbb{N}}$ be sequences such that

$$
x_{0}<b_{n+1}<r_{n}<s_{n}<a_{n}<b_{n}<\beta
$$

for any $n \in \mathbb{N}$ and the right-hand density of the set $A=\bigcup_{n=1}^{\infty}\left[a_{n}, b_{n}\right]$ at $x_{0}$ be equal to 1 .

Firstly, for any $n \in \mathbb{N}$ we will define a function $\tau_{n}$ on each $\left[r_{n}, s_{n}\right]$ by using the same method as in the previous part of the proof. Fix $n \in \mathbb{N}$. Let $a_{1}^{n}, a_{2}^{n}, \ldots, a_{2^{n}}^{n}$ be the points from $\left(r_{n}, s_{n}\right)$ which divide this interval into $2^{n}+1$ closed intervals of equal length. Put

$$
\tau_{n}(x)= \begin{cases}r_{n} & \text { for } x \in\left\{r_{n}\right\} \cup\left\{a_{2 m}^{n}: m=1,2, \ldots, 2^{n-1}\right\}, \\ s_{n} & \text { for } x \in\left\{s_{n}\right\} \cup\left\{a_{2 m-1}^{n}: m=1,2, \ldots, 2^{n-1}\right\}, \\ \text { linear } & \text { on }\left[r_{n}, a_{1}^{n}\right],\left[a_{2^{n}}^{n}, s_{n}\right],\left[a_{i}^{n}, a_{i+1}^{n}\right], i=1, \ldots, 2^{n}-1 .\end{cases}
$$


Define $\tau$ on the set $[\alpha, \beta]$ by the formula

$$
\tau(x)= \begin{cases}x_{0} & \text { for } x \in\left[\alpha, x_{0}\right] \\ x & \text { for } x \in A \\ \tau_{n}(x) & \text { for } x \in\left[r_{n}, s_{n}\right], n \in \mathbb{N}, \\ \text { linear } & \text { on the intervals }\left[b_{1}, \beta\right],\left[b_{n+1}, r_{n}\right],\left[s_{n}, a_{n}\right], n \in \mathbb{N} .\end{cases}
$$

Moreover, we put $\tau(x)=\phi(x)$ on $\mathbb{I} \backslash \operatorname{proj}_{X}(K)$ and linear on $\operatorname{proj}_{X}(K) \backslash[\alpha, \beta]$ so that $\tau$ is continuous on the interval $\mathbb{I}$. Obviously, $\Gamma(\tau) \subset U$.

We shall show that $\tau$ fulfils all conditions from (iii). Observe that $x_{0}$ is an odd point of $\tau$. Indeed, obviously $x_{0} \in \operatorname{Fix}(\tau)$. Let $\varepsilon>0$. There is $n_{0} \in \mathbb{N}$ such that $b_{n_{0}}<$ $x_{0}+\min \left\{\varepsilon, x_{0}-\alpha\right\}$. Put $\delta_{0}=b_{n_{0}}-x_{0}$ and $P=\left[x_{0}-\delta_{0}, x_{0}+\delta_{0}\right] \subset\left(x_{0}-\varepsilon, x_{0}+\varepsilon\right)$. It is easy to see that $\tau^{n}(P) \subset P$ for any $n \in \mathbb{N}$, which gives that $x_{0}$ is an almost stable point of $\tau$.

Moreover, by putting $B_{\tau}^{n}=\left(\mathcal{F}_{n}, J_{n}\right), \mathcal{F}_{n}=\left\{\left[a_{2 i-1}^{n}, a_{2 i}^{n}\right]: i=1, \ldots, 2^{n-1}\right\}$ and $J_{n}=\left[r_{n}, s_{n}\right]$, we can show, analogously to in the first part of the proof, that the entropy of $\tau$ at $x_{0}$ is infinite, which completes the proof of (A1).

To show condition $\left(\mathrm{B} 1^{\prime}\right)$, consider sequences $\left\{z_{n}\right\}_{n \in \mathbb{N}},\left\{t_{n}\right\}_{n \in \mathbb{N}} \subset\left(\alpha, x_{0}\right)$ convergent to $x_{0}$ such that $z_{n}<t_{n}<z_{n+1}$ for $n \in \mathbb{N}$ and the left-hand density of $\bigcup_{n=1}^{\infty}\left[z_{n}, t_{n}\right]$ at $x_{0}$ is equal to 1 . Obviously, for $A=\bigcup_{n=1}^{\infty}\left[z_{n}, t_{n}\right] \cup\left\{x_{0}\right\} \cup \bigcup_{n=1}^{\infty}\left[a_{n}, b_{n}\right]$ we obtain that $A \in \mathcal{L}_{i}\left(x_{0}\right)$ and $x_{0}$ is a density point of $A$. Moreover, we see at once that $\lim _{A \ni x \rightarrow x_{0}} \tau(x)=\tau\left(x_{0}\right)$. Since $\tau$ is constant on $\bigcup_{n=1}^{\infty}\left[z_{n}, t_{n}\right] \cup\left\{x_{0}\right\}$ and $\tau(x)=x$ for $x \in \bigcup_{n=1}^{\infty}\left[a_{n}, b_{n}\right]$ we have that $h(\tau, A)=0$.

Similarly, like in the previous case we can prove that $\tau$ satisfies condition $(\mathrm{C} 1)$ (for any $\varepsilon>0$ we will use some interval $\left[r_{n}, s_{n}\right]$ contained in $\left(x_{0}-\varepsilon, x_{0}+\varepsilon\right)$ and divided into $2^{n}+1$ parts each of length $\delta$ ). The proof of implication (i) $\Rightarrow$ (iii) is thus complete.

Open Access This article is distributed under the terms of the Creative Commons Attribution 4.0 International License (http://creativecommons.org/licenses/by/4.0/), which permits unrestricted use, distribution, and reproduction in any medium, provided you give appropriate credit to the original author(s) and the source, provide a link to the Creative Commons license, and indicate if changes were made.

\section{References}

1. Čiklová, M.: Dynamical systems generated by functions with $G_{\delta}$ graphs. Real Anal. Exchange 30(2), 617-637 (2004/2005)

2. Elaydi, S., Sacker, R.J.: Global stability of periodic orbits of non-autonomous difference equations and population biology. J. Differential Equations 208(1), 258-273 (2005)

3. Li, J., Ye, X.D.: Recent development of chaos theory in topological dynamics. Acta Math. Sinica (Engl. Ser.) 32(1), 83-114 (2016)

4. Loranty, A., Pawlak, R.J.: On some sets of almost continuous functions which locally approximate a fixed function. Tatra Mt. Math. Publ. 65, 105-118 (2016)

5. Loranty, A., Pawlak, R.J.: On functions attracting positive entropy. Bull. Aust. Math. Soc. 97(1), 69-79 (2018) 
6. Luis, R., Elaydi, S., Oliveira, H.: Non-autonomous periodic systems with Allee effects. J. Difference Equ. Appl. 16(10), 1179-1196 (2010)

7. Oxtoby, J.C.: Measure and Category. Graduate Texts in Mathematics, vol. 2, 2nd edn. Springer, New York (1980)

8. Pawlak, R.J., Loranty, A.: Stable, almost stable and odd points of dynamical systems. Bull. Aust. Math. Soc. 96(2), 245-255 (2017)

9. Pawlak, R.J., Loranty, A., Bạkowska, A.: On the topological entropy of continuous and almost continuous functions. Topology Appl. 158(15), 2022-2033 (2011)

10. Ruette, S.: Chaos on the Interval. University Lecture Series, vol. 67. American Mathematical Society, Providence (2017). arXiv:1504.03001v2

11. Stallings, J.: Fixed point theorem for connectivity maps. Fund. Math. 47, 249-263 (1959)

12. Yakubu, A.A., Castillo-Chavez, C.: Interplay between local dynamics and dispersal in discrete-time metapopulation models. J. Theoret. Biol. 218(3), 273-288 (2002) 